\title{
Black phosphorus integrated fiber grating for ultrasensitive heavy metal
} sensing

Liu, Chen; Sun, Z.; Zhang, L.; Lv, J.; Yu, X.; Zhang, L.; Chen, Xianfeng

\section{Sensors and Actuators B: Chemical}

DOI:

10.1016/j.snb.2017.11.022

Published: 01/03/2018

Peer reviewed version

Cyswllt i'r cyhoeddiad / Link to publication

Dyfyniad o'r fersiwn a gyhoeddwyd / Citation for published version (APA):

Liu, C., Sun, Z., Zhang, L., Lv, J., Yu, X., Zhang, L., \& Chen, X. (2018). Black phosphorus integrated fiber grating for ultrasensitive heavy metal sensing. Sensors and Actuators $B$ : Chemical, 257, 1093-1098. https://doi.org/10.1016/j.snb.2017.11.022

\footnotetext{
Hawliau Cyffredinol / General rights

Copyright and moral rights for the publications made accessible in the public portal are retained by the authors and/or other copyright owners and it is a condition of accessing publications that users recognise and abide by the legal requirements associated with these rights.

- Users may download and print one copy of any publication from the public portal for the purpose of private study or research.

- You may not further distribute the material or use it for any profit-making activity or commercial gain

- You may freely distribute the URL identifying the publication in the public portal ?
}

Take down policy

If you believe that this document breaches copyright please contact us providing details, and we will remove access to the work immediately and investigate your claim. 


\title{
Black phosphorus integrated tilted fiber grating for ultrasensitive heavy metal sensing
}

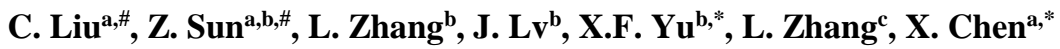 \\ ${ }^{a}$ School of Electronic Engineering, Bangor University, Bangor LL57 1UT, United Kingdom \\ ${ }^{\mathrm{b}}$ Shenzhen Institutes of Advanced Technology, Chinese Academy of Sciences, Shenzhen 518055, P. R. China \\ ${ }^{\mathrm{c}}$ Aston Institute of Photonic Technologies, Aston University, Birmingham B4 7ET, United Kingdom
}

\begin{abstract}
We report the sub-parts-per-billion (sub-ppb) level detection of lead using black phosphorus (BP) integrated tilted fiber grating (BP-TFG) configuration where the light-matter interaction is enhanced by BP nanosheets coating. We synthesized BP nanosheets by using a liquid-phase exfoliation method and developed an in-situ layer-by-layer (i-LbL) technique for BP deposition. These approaches secured high-quality BP coating on specific fiber cylindrical surface with strong adhesion as well as a prospective thickness control. By taking advantage of $\mathrm{i}-\mathrm{LbL}$ deposition, the unique optical tunable features including coating thickness-dependent polarization and polarization-selective coupling have been experimentally observed. Furthermore, the BP-TFG was exploited as the first BP-fiber optic chemical sensor for heavy metal $\mathrm{Pb}^{2+}$ ions detection, demonstrating significant performance of ultrahigh sensitivity up to $0.5 \times 10^{-3} \mathrm{~dB} / \mathrm{ppb}$, much lower limit of detection down to $0.25 \mathrm{ppb}$, and extremely wide concentration range from $0.1 \mathrm{ppb}$ to $1.5 \times 10^{7} \mathrm{ppb}$. We believe that this BP-fiber optic architecture opens the path as an optical platform for superior chemical sensing and biomedical applications.
\end{abstract}

Keywords: Black phosphorus; Nanomaterials; Fiber grating; Optical sensor; Chemical sensor; Heavy metal.

\section{Introduction}

Two-dimensional (2D) layered materials, which include graphene, transition metal dichalcogenides (TMDs), and boron/carbon nitride, have attracted significant attentions [1-4]. Due to their exceptional properties of large surface-to-volume ratio, atomic-thin layer structure and high carrier mobility, 2D materials show a wide range of applications in electronics, light processing, energy storages, and chemical and biochemical sensors [5-8]. Very recently, black phosphorus (BP) sparks intense interest of scientific communities in physics, chemistry, materials, and biology [9-16]. BP has a puckered lattice configuration along the armchair direction and a bilayer structure along the zigzag direction [11]. Thanks to the unique puckered orthorhombic structure and in-plane anisotropy, BP possesses much larger surface-to-volume ratio, extremely high hole mobility and superior molecular adsorption energy than those of other 2D materials $[9,15,16]$. The extraordinary properties of BP make it a very promising nanomaterial for widespread applications such as BP field-effect transistors (FETs), modulators, photodetectors, chemical sensors, bioimaging and biomedical therapy [9-11,16-20].

The novel lab-on-fiber technology has been proposed for optical sensing and optical communications [21,22]. Besides

\# C. Liu and Z. Sun contributed equally to this work.

* Corresponding authors. Tel: +44 1248382480.

Email address: x.chen@bangor.ac.uk (X.Chen).xf.yu@siat.ac.cn (X.F. Yu). the inherent merits of optical fiber, such as compact size, light weight, and electromagnetic interference immunity, in-fiber grating technology provides advantages of real-time, multiplex, high sensitivity, label-free, and in-line determination [23-28]. Both long period fiber grating (LPG) and tilted fiber grating (TFG) can couple the light from fiber core to the cladding yielding an evanescent wave penetrating outside of the cladding. As the evanescent wave can be affected by changes in concentration or composition of the surrounding medium or by molecular interactions occurring on the cladding surface, it is a good candidate for chemical and biochemical sensing [23-24]. In contrast to conventional LPG, TFG owns narrow band resonances demonstrating much higher Q-factor hence high accuracy in optical signal detection [25-28]. In particular, the excessively tilted fiber grating shows much lower thermal and tension cross-talk effects and higher sensitivity in lower refractive index (RI) region [25].

Techniques have been developed to improve the sensitivity of fiber devices by fiber tapering, cladding etching and side polishing. Instead of sacrificing the mechanical integrity, thin film deposition is an alternative elegant approach. To date, various materials including carbon nanotubes (CNTs), titanium dioxide, zinc oxide, and graphene have been employed to enhance the RI sensitivity of fiber sensors [29-32]. However, the grand challenge of film deposition on optical fiber is from its cylindrical geometry and such a thin diameter. The recent exploitation reveals that 2D layered materials could modulate light with superior performance for realistic applications in 
ultrafast lasers, broadband polarizer, gas detection, humidity sensor, and biosensing [6,7,33-36].

Heavy metals are usually considered to be toxic and carcinogenic with the harmful effects on the environment, drinking-water and living organisms. It needs to be recognized that lead is exceptional compared with other chemical hazards. Exposure to lead is associated with a wide range of effects, including various neuro developmental effects, cardiovascular diseases, impaired renal function, hypertension, and impaired fertility. According to the guidelines for drinking-water quality of World Health Organization (WHO), the maximum permissible limit of lead in drinking-water is $10 \mathrm{ppb}$ [37], which represents the lead concentration does not exceed tolerable risk to the health. Therefore, the detection of heavy metal becomes a major public concern in modern days.



Fig. 1 Schematic of BP-TFG configuration as an optical tunable platform for strong light-matter interaction.

In this work, we report an integrated BP-fiber optic configuration as an optical tunable platform for the enhancement of light-matter interaction and heavy metal detection. As the schematic illustration shown in Fig. 1, the light passes down the core of a fiber and encounters a $81^{\circ}$-TFG, certain wavelength of the light are forward-coupled into the cladding yielding an evanescent wave penetrating into BP coating, demonstrating strong light-matter interaction. We synthesized BP nanosheets by using a liquid-phase exfoliation method and developed an in-situ layer-by-layer (i-LbL) deposition technique. These approaches secured high-quality
BP overlay deposited on specific cylindrical surface of fiber with strong adhesion as well as a prospective thickness control. The unique optical tunable properties have been experimentally observed. Furthermore, the BP-TFG was exploited as the first BP-fiber chemical sensor for heavy metal detection with significant performance.

\section{Materials and methods}

\subsection{Fabrication of $81^{\circ}$-tilted fiber grating}

The $81^{\circ}$-TFG with length of $12 \mathrm{~mm}$ was UV-inscribed in hydrogen loaded Corning SMF-28 fiber by using a frequency-doubled Ar laser and mask scanning technique. A custom-designed mask with a pitch of $6.6 \mu \mathrm{m}$ was purchased from Edmund Optics Ltd. The mask was rotated in the plane of incidence to realize grating fringes tilted by $81^{\circ}$ with respect to the fiber axis. Multiple scans were applied to achieve a maximum forward cladding mode coupling at the wavelength determined by the following phase-matching condition:

$$
\lambda_{c o-c l}=\left(n_{c o, i}^{e f f}-n_{c l, m}^{e f f}\right) \cdot \frac{\Lambda_{g}}{\cos \theta}
$$

where $n_{c o, i}^{e f f}$ is the effective index of fiber core, $i$ represents transverse-magnetic (TM) or transverse-electric (TE) polarization state, $n_{c l, m}^{e f f}$ is the effective index of cladding, $m$ means $m$ th mode, $\Lambda_{g}$ is the normal period of grating, and $\theta$ is the tilted angle.

\subsection{Synthesis of BP nanosheets}

The BP nanosheets were synthesized by using a liquid-phase exfoliation method, which was similar to our previous work [20], except the solvent in this work was changed with absolute ethanol. As shown in Fig. 2: (i) $25 \mathrm{mg}$ bulk BP was cut and grinded into small pieces then added to $25 \mathrm{~mL}$ absolute ethanol; (ii) The BP dispersion was sonicated by a cell crasher at $25 \mathrm{kHz}$ and $1200 \mathrm{~W}$ for $3 \mathrm{~h}$ to break the van-der Waals stack of BP, where the temperature of solution was kept below $277 \mathrm{~K}$ with an ice bath; (iii) The as-prepared dispersion was centrifuged at $5000 \mathrm{rpm}$ for $15 \mathrm{~min}$; (iv) the supernatant containing few-layered BP nanosheets was decanted gently. (i)



(ii)

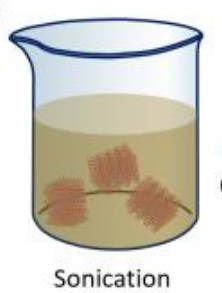

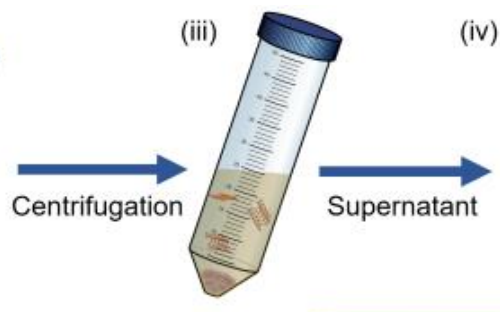

(iv)

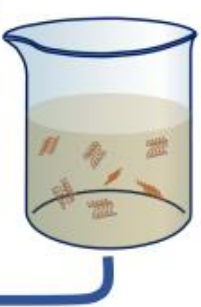

(vii)

(v)

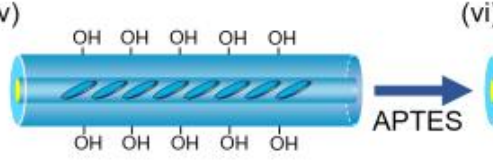

(vi)

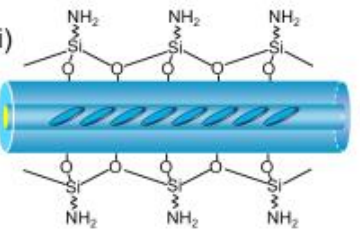

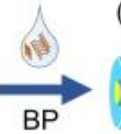

BP

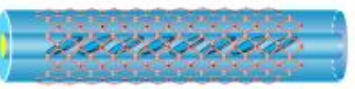

Fig. 2 Schematic representation of synthesis of BP nanosheets (i-iv) and deposition process of BP nanosheets on fiber device surface (v-vii). 


\subsection{BP deposition on fiber surface}

For BP deposition, we developed an i-LbL technique, which was based on chemical surface modification in conjunction with in-situ layer-by-layer assembly. The deposition process consists of: (v) The section of fiber over TFG region was rinsed with acetone to clean the surface from any contaminants. Thereafter, an alkaline treatment was carried out by immersing the fiber device in $1.0 \mathrm{M} \mathrm{NaOH}$ solution for $1 \mathrm{~h}$ to enrich the number of -OH groups on the fiber surface, then washed with DI water and ethanol thoroughly; (vi) A silanization procedure was followed by incubating the fiber in freshly-prepared 5\% (3Aminopropyl)triethoxysilane (APTES) for $2 \mathrm{~h}$ to form $\mathrm{Si}-\mathrm{O}-\mathrm{Si}$ bonding; (vii) The fiber was fixed into a homemade microchannel container where $30 \mu \mathrm{L}$ BP dispersion was carefully added. Due to positively-charged amino groups on the APTES-silanized fiber surface, the negatively-charged BP nanosheets were adhered to fiber by electrostatic force. After ethanol solvent fully evaporated, the fiber surface was dried and coated with BP nanosheets (Fig. S1). Subsequently, the second deposition cycle was conducted. In each cycle, BP dispersion (30 $\mu \mathrm{L}$ ) was added in microchannel container where ethanol solvent was evaporating gradually and totally, such drying process enhanced the physisorption of BP nanosheets on fiber surface. After 8 cycles, the BP-coated TFG was rinsed by absolute ethanol to remove non-adhered BP nanosheets, dried and stored in a vacuum environment to enhance the layer-by-layer adhesion before further uses. The transmission spectra of BP-TFG were monitored in real-time during the entire $\mathrm{BP}$ deposition process, which will be discussed later.

(a)

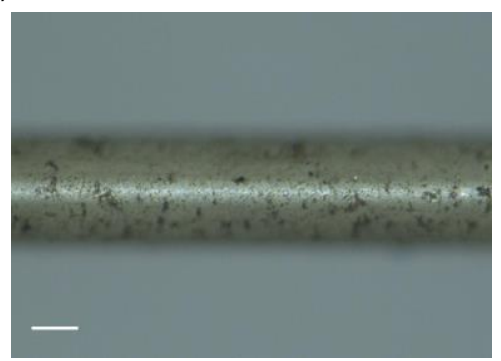

(b)

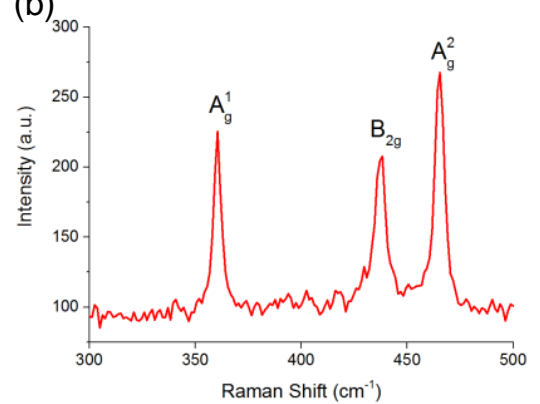

(c)

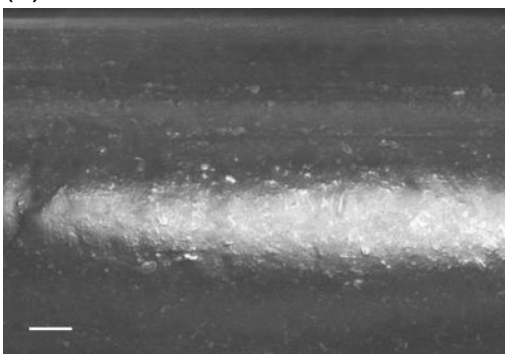

(d)

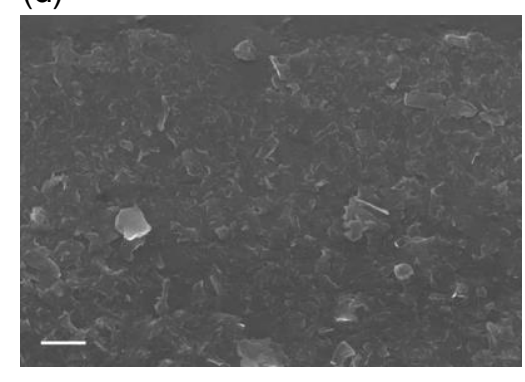

\section{Results and discussion}

\subsection{Surface morphological characterization}

The surface morphological characterization was investigated by using digital microscope (Keyence VHX-2000), Raman spectroscopy (Horiba Jobin-Yvon Lab Ram HR VIS, 633 nm), scanning electron microscope (SEM, Zeiss SUPRA 55), and atomic force microscopy (AFM, Veeco Instruments Inc.).

The initial microscope image Fig. 3(a) shows a successful overlay coated on fiber surface. Subsequently, Raman spectroscopy was used to characterize the coating material. Raman spectrum in Fig. 3(b) presents three characteristic peaks, which include $\mathrm{A}_{\mathrm{g}}^{1}$ (at $\left.\sim 362 \mathrm{~cm}^{-1}\right), \mathrm{B}_{2 \mathrm{~g}}\left(\right.$ at $\sim 440 \mathrm{~cm}^{-1}$ ) and $\mathrm{A}_{\mathrm{g}}^{2}$ (at $\sim 466 \mathrm{~cm}^{-1}$ ), indicating the presence of black phosphorus [11]. The $\mathrm{A}_{\mathrm{g}}{ }_{\mathrm{g}}$ corresponds to atoms oscillate out-of-plane while $\mathrm{B}_{2 \mathrm{~g}}$ and $\mathrm{A}_{\mathrm{g}}^{2}$ correspond to atoms vibrate within the plane. The surface coverage of BP was further examined by SEM with the magnification of $1000 \times$ (Fig. 3c) and 10000× (Fig. 3d). It can be seen that the BP nanosheets are densely covered on the fiber and BP overlay is quite homogeneous over the entire cylindrical surface demonstrating the high-quality BP deposition.

The thickness of BP overlay depends on the conditions of deposition. With the flexibility of i-LbL deposition technique, the overlay thickness can be prospectively controlled by the number of coating cycles. Fig. 3(e) and Fig. 3(f) plot AFM tapping mode topographic images of BP overlay on fiber surface after 8 coating cycles, in which the thickness of BP overlay was measured as $\sim 323.1 \mathrm{~nm}$.

Fig. 3 Surface morphology of BP-coated fiber. (a) Microscope image (scale bar: $50 \mu \mathrm{m}$ ), (b) Raman spectrum, SEM images with magnification of (c) $1000 \times($ bar: $10 \mu \mathrm{m}$ ) and (d) 10000× (bar: $1 \mu \mathrm{m}$ ), Atomic force micrograph topographic (e) 3D image and (f) profile of BP coating boundary (inset: height profile of BP overlay). 


\subsection{Observation of optical tunable properties}

The optical properties of BP-TFG were experimentally investigated by monitoring BP-TFG transmission spectra over entire BP deposition process. For a $81^{\circ}$-TFG, the excessively tilted plane of index-modulated grating breaks the symmetry of mode coupling, inducing the significant polarization dependent coupling characteristics. $81^{\circ}$-TFG exhibits two sets of comb-like split resonances in transmission spectra, which suggest that the light have been coupled into two sets of birefringence modes corresponding to two orthogonal polarization modes, TM and TE modes [27]. The polarization modes can be fully excited by launching the linearly polarized light. Spectra (i) in Fig. 4(a) show a zoomed-pair of polarization modes, in which TM and TE modes have been fully excited, respectively. The transmission spectra of BP-TFG were monitored during deposition process and recorded as (ii), (iii), (iv) and (v) in Fig. 4(a) after $2^{\text {nd }}, 4^{\text {th }}, 6^{\text {th }}$ and $8^{\text {th }}$ coating cycle, respectively.
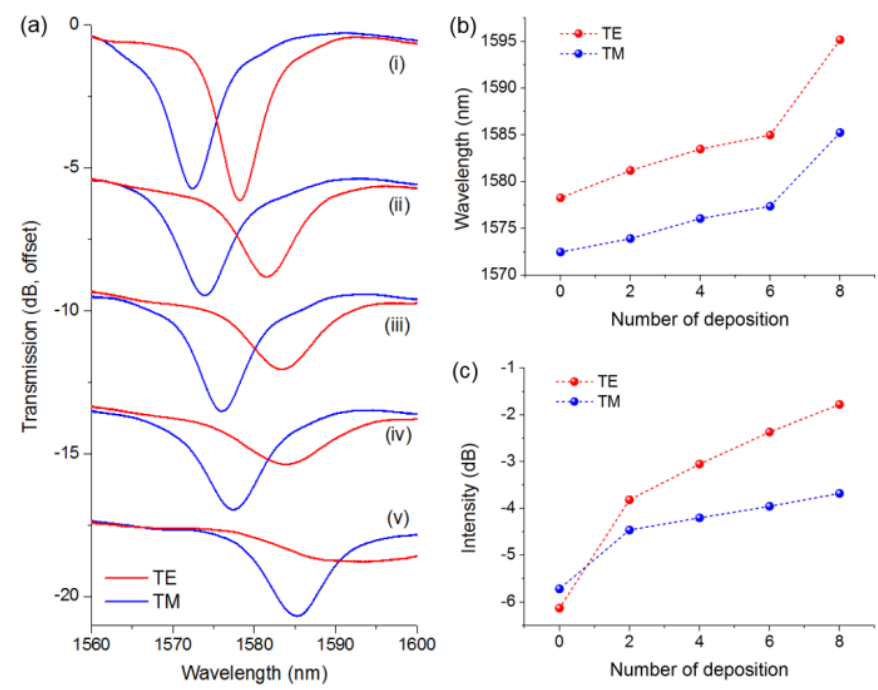

Fig. 4 (a) Spectral evolution during BP-deposition process revealing strong light-matter interface and optical tunable features. (b) Wavelength shift and (c) intensity change of TM- and TE-polarized resonances against number of deposition cycles.

It is apparent that the polarization depends on BP overlay thickness. As can be seen from Fig. 4, optical signals (both TM- and TE-polarized resonances) show a significant red-shift in wavelength and a decrease in intensity against the increase of overlay thickness which was determined by number of coating cycles. The wavelength shift was attributed to the increase of cladding effective refractive index induced by high RI coating layer, and consequently the phase-matching condition Eq. (1) was tuned. The leaky radiation from cladding to coating layer could reduce the coupling coefficient between core and cladding modes hence decrease the resonant intensity.

It should be emphasized that the extinction ratio has been extremely enhanced with the increase of BP overlay thickness, meanwhile a polarization-selective coupling feature has been clearly observed. As shown in Fig. 4, the variation of TE mode is always larger than that of TM mode. After 8 cycles of BP deposition, TE mode has $16.9 \mathrm{~nm}$ in wavelength shift (Fig. 4b) and $4.35 \mathrm{~dB}$ in intensity decrease (Fig. 4c), which are 1.2 times and 2.1 times larger than those of TM mode, respectively. The separation between two polarized resonances of BP-TFG has been increased to $8.38 \mathrm{~nm}$, giving an estimated birefringence of $\sim 3.0 \times 10^{-4}$ which is $150 \%$ enhancement that of bare $81^{\circ}$-TFG. In such BP-TFG architecture, TE-polarized radiative light can be strongly perturbed by BP overlay in terms of coupling and guiding, leaving the TM-polarized light passes through the fiber.

\subsection{Strong ability of $\mathrm{Pb}^{2+}$ adsorption and ultrahigh sensitivity}

The BP-TFG was exploited for the implementation of BP strong ability of lead ions adsorption and ultrahigh sensitivity for heavy metal chemical sensing.

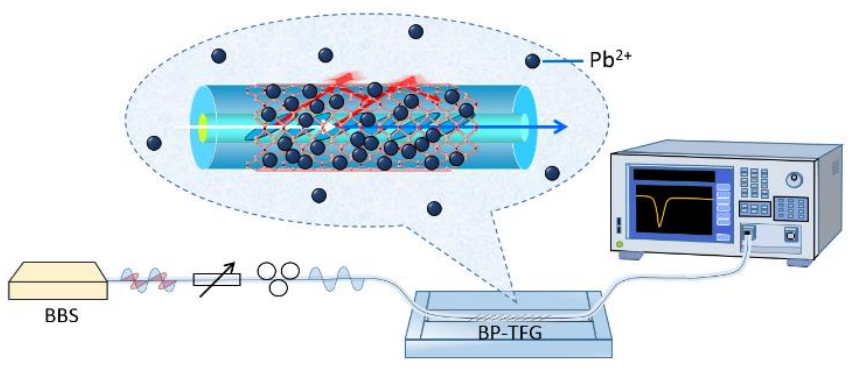

Fig. 5 Experiment setup for heavy metal chemical sensing.

The experiments were conducted with the setup illustrated in Fig. 5. The light from broadband source was launched into BP-TFG and the output was monitored by an optical spectrum analyzer (OSA). By adjusting the polarization controller, the TM-polarized resonance was fully excited and selected for optical signal monitoring. To avoid thermal and tension cross-talk effects, the BP-TFG was mounted on a homemade mini-bath with two ends fixed and all the experiments were implemented at room temperature. A series of aqueous solutions containing $\mathrm{Pb}^{2+}$ ions with concentrations of $0.1,1.0,10,100,1000$, $1 \times 10^{4}, 1 \times 10^{5}, 1 \times 10^{6}$ and $1.5 \times 10^{7} \mathrm{ppb}$ were prepared for the detection. The BP-TFG was firstly immersed into $\mathrm{Pb}^{2+}$ solution for $120 \mathrm{~s}$, the solution was subsequently withdrawn by careful pipetting to expose the BP-TFG in the air for $180 \mathrm{~s}$ when the transmission spectrum achieved steady and was captured by OSA. Afterwards, the BP-TFG was washed with ethanol thoroughly to remove the adsorbed lead ions prior to next concentration measurement.

The transmission spectra at different $\mathrm{Pb}^{2+}$ concentrations are plotted in Fig. 6(a). By increasing $\mathrm{Pb}^{2+}$ concentration, the peak shows apparent intensity decrease indicating the strong optical absorption occurred between BP and lead ions. The slight wavelength red-shift could be caused by the increase of effective refractive index of cladding. Fig. 6(b) depicts the intensity change as a function of ion concentrations, revealing a non-linear relationship with piecewise sensitivities of $0.5 \times 10^{-3} \mathrm{~dB} / \mathrm{ppb}, 7.7 \times 10^{-7} \mathrm{~dB} / \mathrm{ppb}$ and $2.3 \times 10^{-8}$ $\mathrm{dB} / \mathrm{ppb}$ for $\mathrm{Pb}^{2+}$ concentration ranges of $0 \sim 100 \mathrm{ppb}, 10^{3} \sim 10^{5} \mathrm{ppb}$, and $10^{6} \sim 10^{7} \mathrm{ppb}$, respectively. The proposed BP-TFG can effectively detect $\mathrm{Pb}^{2+}$ ions concentration over a wide range from $0.1 \mathrm{ppb}$ to $1.5 \times 10^{7} \mathrm{ppb}$ with no saturation effect, which is four orders of magnitude larger than that of BP-FET based lead sensor [17]. The 
reported BP-FET sensor limited its detection for $\mathrm{Pb}^{2+}$ concentrations lower than $10 \mathrm{ppb}$ and faced the saturation problem for concentrations higher than $1 \times 10^{5} \mathrm{ppb}$.

(a)

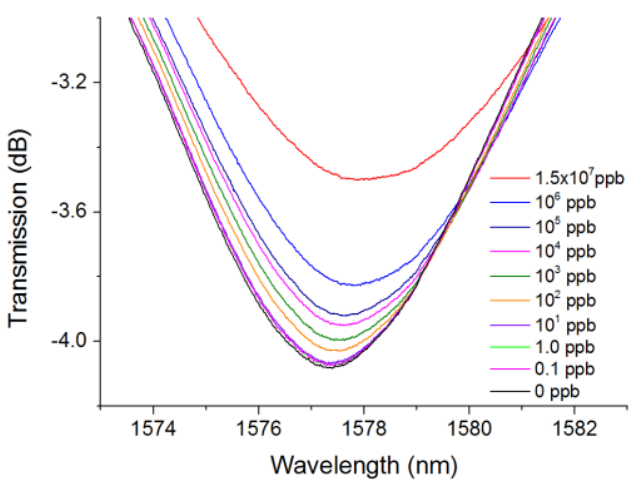

(b)

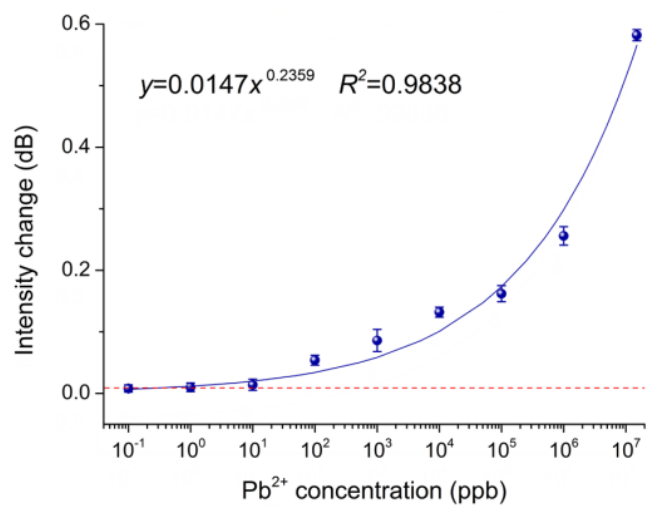

Fig. 6 (a) Transmission spectra of TM-resonance showing a clear up-shift as $\mathrm{Pb}^{2+}$ concentration increases. (b) Resonant intensity change against $\mathrm{Pb}^{2+}$ concentrations. The blue curve is the best logistic fitting line and the dashed horizontal red line represents three times the standard deviation of the blank measurement.

The limit of detection (LOD) is generally defined as three times the standard deviation of its blank measurement. The blue curve in Fig. 6(b) provides the best logistic fitting of the experimental data, whereas the dashed red line represents intensity change three times the standard deviation. Therefore, the LOD of BP-TFG is $0.25 \mathrm{ppb}$ for $\mathrm{Pb}^{2+}$ ions, which is 4-fold better than CNTs functionalized heavy metal sensor [38]. Meanwhile, this LOD of $0.25 \mathrm{ppb}$ is only one fortieth of WHO's permissible limit of $10 \mathrm{ppb}$ for lead in drinking-water [37]. For comparison, the similar detection was also conducted for light metal $\mathrm{Na}^{+}$ions with the results plotted as green symbols in Fig. S2, showing a very limited sensitivity. It reveals that BP exhibits strong adsorption of heavy metal ions hence the ultrahigh sensitivity for lead detection. In addition, the proposed BP-TFG has been demonstrated its excellent reusability for lead detection (Fig. S3), showing the potential to develop a more cost-effective and reusable chemical sensor.

\subsection{Heavy metal adsorption-induced optical signal change}

We discuss the basic mechanism underpinning the unique optical properties of BP-TFG with the adsorption of heavy metal ions. It has been reported that BP band gap can be widely tunable by thickness, strain, electric field and dopant [12-14]. As the pristine BP behaves as a p-type semiconducting characteristic, its majority charge carriers are holes. The adsorption of heavy metal ions denoted charges and enhanced hole carrier concentration in BP. With increasing ion density, the electric field from the ionized donors gradually reduced band gap owing to the giant Stark effect and tuned the electronic state of BP from a semiconductor to a Dirac semimetal [12]. Due to its ultrahigh carrier mobility and superior molecular adsorption energy, BP has strong capability to attract heavy metal ions, which is more efficient for charge transfer between BP and heavy metal ions. When the carrier concentration was large enough, correlation effects became significant and resulted in an increase of carrier-induced refractive index change. Consequently, the fiber guided wave was modulated by perturbing phase condition and optical absorption coefficient.

\section{Conclusions}

In conclusion, we have reported an integrated BP-TFG configuration for enhancement of light-matter interface and for chemical sensing. The developed i-LbL deposition technique secured high-quality BP overlay and prospective thickness control. We have experimentally observed the unique optical properties including overlay thickness-dependent polarization and polarization-selective coupling. With BP deposition, both phase condition and light coupling coefficient of TFG have been modulated with the corresponding resonant wavelength shift and intensity change. The BP-TFG has been implemented for $\mathrm{Pb}^{2+}$ detection, demonstrating ultrahigh sensitivity up to $0.5 \times 10^{-3} \mathrm{~dB} / \mathrm{ppb}$, much lower LOD of 0.25 $\mathrm{ppb}$, and extremely wide concentration range from $0.1 \mathrm{ppb}$ to $1.5 \times 10^{7}$ ppb. The BP-TFG based sensor has achieved a sub-ppb level detection of $\mathrm{Pb}^{2+}$ in water. In addition, the wide detection range is 4-order of magnitude larger than that of BP-FET based lead sensor [17]. The proposed BP-fiber optic architecture provides a remarkable platform for chemical and biochemical applications.

\section{Acknowledgements}

This work was supported by the EU FP7 PIRSES-GA-2013-612267 (iPhoto-Bio), the Sêr Cymru NRN Fund (NRN097), and National Natural Science Foundation of China (No. 51672305, No. 81501577).

\section{References}

[1] K. S. Novoselov, A. K. Geim, S. V. Morozov, D. Jiang, Y. Zhang, S. V. Dubonos, I. V. Grigorieva, and A. A. Firsov, "Electric field effect in atomically thin carbon films," Science 306, 666-669 (2004).

[2] A. K. Geim, "Graphene: status and prospects," Science 324, 1530-1534 (2009).

[3] X. Huang, Z. Zeng, and H. Zhang, "Metal dichalcogenide nanosheets: preparation, properties and applications," Chem. Soc. Rev. 42, 1934-1946 (2013).

[4] Z. Sun, A. Martinez, and F. Wang, "Optical modulators with 2D layered materials," Nature Photon. 10, 227-238 (2016).

[5] F. Bonaccorso, Z. Sun, T. Hasan, and A. C. Ferrari, "Graphene photonics and optoelectronics," Nature Photon. 4, 611-622 (2010).

[6] Q. Bao, H. Zhang, Y. Wang, Z. H. Ni, Y. L. Yan, Z. X. Shen, K. P. Loh, and D. Y. Tang, "Atomic-layer graphene as a saturable absorber for ultrafast pulsed lasers," Adv. Funct. Mater. 19, 3077-3083 (2009).

[7] Q. Bao, H. Zhang, B. Wang, Z. Ni, C. H. Y. X. Lim, Y. Wang, D. Y. Tang, and K. P. Loh, "Broadband graphene polarizer," Nat. Photon., 5, 411-415 (2011).

[8] K. P. Loh, Q. Bao, G. Eda, and M. Chhowalla, "Graphene oxide as a chemically tunable platform for optical applications" Nat. Chem. 2, 1015-1024 (2010). 
[9] L. Li, Y. Yu, G. J. Ye, Q. Ge, X. Ou, H. Wu, D. Feng, X. H. Chen, and Y. Zhang, "Black phosphorus field-effect transistors," Nat. Nanotechnol. 9, 372-377 (2014).

[10]F. Xia, H. Wang, and Y. Jia, "Rediscovering black phosphorus as an anisotropic layered material for optoelectronics and electronics," Nat. Commun. 5, 4458 (2014).

[11] A. Castellanos-Gomez, "Black phosphorus: narrow gap, wide applications," J. Phys. Chem. Lett. 6, 4280-4291 (2015).

[12] J. Kim, S. S. Baik, S. H. Ryu, Y. Sohn, S. Park, B. G. Park, J. Denlinger, Y. Yi, H. J. Choi, and K. S. Sim, "Observation of tunable band gap and anisotropic Dirac semimetal state in black phosphorus," Science 349, 723-726 (2015).

[13] A. S. Rodin, A. Carvalho, and A. H. Castro Neto, "Strain-induced gap modification in black phosphorus," Phys. Rev. Lett. 112, 176801 (2014).

[14] R. Fei and L. Yang, "Lattice vibrational modes and Raman scattering spectra of strained phosphorene," Appl. Phys. Lett. 105, 083120 (2014).

[15] H. Liu, A. T. Neal, Z. Zhu, X. Xu, D. Tomanek, and P. D. Ye, "Phosphorene: an unexplored 2D semiconductor with a high hole mobility," ACS Nano 8, 4033-4041 (2014)

[16] S. Cho, Y. Lee, H. Koh, H. Jung, J. Kim, H. Yoo, J. Kim, and H. Jung, "Superior chemical sensing performance of black phosphorus: comparison with $\mathrm{MoS}_{2}$ and graphene," Adv. Mater. 28, 7020-7028 (2016).

[17] P. Li, D. Zhang, J. Liu, H. Chang, Y. Sun, and N. Yin, "Air-stable black phosphorus devices for ion sensing," ACS Appl. Mater. Interfaces 7, 24396-24402 (2015)

[18] A. N. Abbas, B. Liu, L. Chen, Y. Ma, S. Cong, N. Aroonyadet, M. Köpf, T. Nilges, and C. Zhou, "Black phosphorus gas sensors," ACS nano 9, 5618-5624 (2015).

[19] J. Shao, H. Xie, H. Huang, Z. Li, Z. Sun, Y. Xu, Q. Xiao, X.-F. Yu, Y. Zhao, H. Zhang, H. Wang, and P. K. Chu, "Biodegradable black phosphorus-based nanospheres for in vivo photothermal cancer therapy," Nat. Commun. 7, 12967 (2016).

[20] Z. Sun, H. Xie, S. Tang, X. F. Yu, Z. Guo, J. Shao, H. Zhang, H. Huang, H Wang, and P. K. Chu, "Ultrasmall black phosphorus quantum dots: synthesis and use as photothermal agents," Angew. Chem. 127, 11688-11692 (2015).

[21] A. Cusano, M. Consales, A. Crescitelli, and A. Ricciardi, (Eds.) Lab-on-Fiber Technology. Springer (2014).

[22] X. D. Wang and O. S. Wolfbeis, "Fiber-optic chemical sensors and biosensors (2008-2012)," Anal Chem. 85, 487-508 (2013).

[23] T. Erdogan, "Cladding-mode resonances in short- and long-period fiber grating filters," J. Opt. Soc. Am. A 14, 1760-1773 (1997).

[24] S. W. James and R. P. Tatam, "Optical fibre long-period grating sensors: characteristics and application," Meas. Sci. Technol. 14, R49-R61 (2003).

[25] K. Zhou, L. Zhang, X. Chen, and I. Bennion, "Low thermal sensitivity grating devices based on ex $-45^{\circ}$ tilting structure capable of forward-propagating cladding modes coupling," J. Lightw. Technol. 24, 5087-5094 (2006).

[26] J. Ablert, L. Y. Shao, and C. Caucheteur, "Tilted fiber Bragg grating sensors," Laser Photonics Rev. 7, 83-108 (2013).

[27] B. Luo, Z. Yan, Z. Sun, J. Li, and L. Zhang, "Novel glucose sensor based on enzyme-immobilized $81^{\circ}$ tilted fiber grating," Opt. Express 22, 30571-30578 (2014)

[28] C. Caucheteur, T. Guo, F. Liu, B. Guan, and J. Ablert, "Ultrasensitive plasmonic sensing in air using optical fibre spectral combs," Nat. Commun. 7, 13371 (2016).

[29] Y. C. Tan, W. B. Ji, V. Mamidala, K. K. Chow, and S.C. Tjin, "Carbon-nanotube-deposited long period fiber grating for continuous refractive index sensor applications," Sens. Actuators B 196, 260-264 (2014).

[30] L. Coelho, D.Viegas, J.L. Santos, and J.M.M.M. de Almeida, "Enhanced refractive index sensing characteristics of optical fibre long period grating coated with titanium dioxide thin films," Sens. Actuators B 202, 929-934 (2014)

[31] L. Coelho, D. Viegas, J.L.Santos, and J.M.M.M. de Almeida, "Characterization of zinc oxide coated optical fiber long period gratings with improved refractive index sensing properties," Sens. Actuators B 223, 45-51 (2016).

[32] B. Jiang, G. Yin, K. Zhou, C. Wang, X. Gan, J. Zhao, and L. Zhang, "Graphene-induced unique polarization tuning properties of excessively tilted fiber grating," Opt. Lett. 41, 5450-5453 (2016).

[33] T. Allsop, R. Arif, R. Neal, K. Kalli, V. Kundrát, A. Rozhin, P. Culverhouse, and D. J. Webb, "Photonic gas sensors exploiting directly the optical properties of hybrid carbon nanotube localized surface plasmon structures," Light Sci. Appl. 5, e16036 (2016).

[34] Y. Wang, C. Shen, W. Lou, F. Shentu, C. Zhong, X. Dong, and L. Tong, "Fiber optic relative humidity sensor based on the tilted fiber Bragg grating coated with graphene oxide," Appl. Phys. Lett. 109, 031107 (2016).

[35] L. Marques, F. U. Hernandeza, S. W. James, S. P. Morgan, M. Clark, R. P. Tatam, and S. Korposh, "Highly sensitive optical fibre long period grating biosensor anchored with silica core gold shell nanoparticles," Biosens Bioelectron. 75, 222-231. (2016)

[36] C. Liu, Q. Cai, B. Xu, W. Zhu, L. Zhang, J. Zhao, and X. Chen, "Graphene oxide functionalized long period grating for ultrasensitive label-free immunosensing," Biosens Bioelectron. 94, 200-206 (2017).

[37] World Health Organization. Guidelines for Drinking-water Quality, 4th ed. (2011).

[38] M. Shaban and A. R. Galaly, "Highly sensitive and selective in-situ SERS detection of $\mathrm{Pb}^{2+}, \mathrm{Hg}^{2+}$, and $\mathrm{Cd}^{2+}$ using nanoporous membrane functionalized with CNTs," Sci. Rep. 6, 25307 (2016).

\section{Biographies}

Chen Liu received his B.Sc. in Physics in 2012 and M.Sc. in Optics in 2015 from Soochow University, China. He is currently pursuing his Ph.D. degree in the School of Electronic Engineering, Bangor University, U.K. His research interests include fiber gratings, optical fiber sensors, chemical and bio- sensing applications.

Zhengbo Sun received his Ph.D. in Microbiology in 2012 from Wuhan Unviersity, China. Since 2012, he has been an Assistant Researcher in Shenzhen Institutes of Advanced Technology, Chinese Academy of Sciences. His research interests include using functional nanomaterials for optical applications in biomedical targeted diagnosis, drug delivery and cancer therapy. He has published 5 papers as first author in Angew. Chem., Small, Biomaterials etc.

Liang Zhang received the B.Sc and Ph.D degrees from Huazhong University of Science and Technology, China, in 2009 and 2014, respectively. He is a postdoctoral fellow in Shenzhen Institutes of Advanced Technology, Chinese Academy of Sciences. His current research focuses on fiber sensors, fiber resonator, and mode-locked fiber lasers.

Jiancheng $\mathbf{L v}$ is Deputy Director of Shenzhen Institutes of Advanced Technology, Chinese Academy of Sciences. He is a professor on optical design, electronic circuitry, mechanical and structural design, and software programming.

Xuefeng Yu received his B.Sc. in Physics (2000) and Ph.D. in Optics (2003) from Wuhan University, China. Currently he is a Professor and Director of Institute of Biomedicine and Biotechnology, Shenzhen Institutes of Advanced Technology, Chinese Academy of Sciences. His main research focuses on the synthesis and biomedical applications of advanced nanomaterials. As a corresponding author, he has published more than 60 papers in Adv. Mater., Nature Commun., Adv. Funct. Mater., Angew. Chem., ACS Nano, Nano Letters, Biomaterials, and Small with total citations more than 2500

Lin Zhang received the $\mathrm{Ph} . \mathrm{D}$ degree in Physics from the University of Sussex, UK in 1990. She joined the Aston Institute of Photonic Technologies in the School of Engineering \& Applied Science at Aston University in 1994, and was appointed to a Lecturer in 2001 and a Professor in 2005. She has been researching in the area of advanced fiber grating devices, technologies and applications and published over 400 papers in peer-reviewed journals and international conferences.

Xianfeng Chen received his Ph.D in Photonics from Aston University, UK in 2007, then he worked there as a Research Associate. In 2012, he joined the faculty in the School of Electronic Engineering at Bangor University where he is currently a Senior Lecturer. His research interests are in optical sensors, fiber gratings, laser micro/nano fabrication, integration of $2 \mathrm{D}$ materials and photonics, biophotonics, biosensing and biomedical applications. He has published more than 130 papers in peer-reviewed journals and international conferences. 